\title{
Georg Wilhelm Pabst : blessure de la guerre, cicatrices de la pellicule
}

\author{
Louise Dumas
}

\section{Abstract}

Von allen Filmemachern, die Deutschlands filmischen Ruhm in der Zwischenkriegszeit ausmachten, ist G. W. Pabst derjenige, der am wenigsten Anerkennung erhielt - oder am längsten dafür gebraucht hat. Er ist aber auch derjenige, der schon Ende der 1920er Jahre den Ersten Weltkrieg zu einem filmischen Ereignis machte, das in vier seiner Filme mehr oder weniger direkt thematisiert wird: Die Liebe der Jeanne Ney, Westfront 1918, Kameradschaft und Mademoiselle Docteur. Der Krieg wird als eine traumatische Erfahrung dargestellt, die Einzelpersonen, Seelen und Völker verwundet hat. Dabei bilden auch diese vier Filme ein Manifest der Filmkunst, die grundsätzlich eine Dialektik vom Trennen und Verbinden ist. In diesem Sinne hat das Kino durch seine medienästhetischen Mittel die Kraft, die Wunden des Krieges zu verarbeiten und zu heilen.

De tous les réalisateurs qui ont fait la gloire cinématographique de l'Allemagne dans l'entre-deux-guerres, G. W. Pabst est celui qui a été le moins reconnu, ou qui a mis le plus de temps à l'être, mais il est aussi celui qui, dès la fin des années 1920, a fait de la Première Guerre mondiale un événement cinématographique que reflètent, plus ou moins directement, quatre de ses films : L'Amour de Jeanne Ney, Quatre de l'infanterie, La Tragédie de la mine et Mademoiselle Docteur. La guerre y apparaît comme une expérience traumatique qui blesse les individus, les âmes et les peuples. Mais ces quatre films forment aussi un manifeste artistique, où Pabst affirme le pouvoir réparateur du cinéma qui, par essence, consiste en une incessante dialectique entre la coupure et la réunion. Avec les moyens qui lui sont propres, le septième art peut ainsi réparer les séquelles de la guerre.

Of all the filmmakers who made up Germany's cinematic glory in the interwar period, G. W. Pabst is the one who received the least recognition or took the longest to do so. But he is also the one who, as early as the late 1920s, made the First World War a cinematic event which is reflected, more or less directly, in four of his films: The Love of Jeanne Ney, Westfront 1918, Kameradschaft and Street of Shadows. War appears as a traumatic experience that has wounded individuals, souls and peoples. But these four films also form an artistic manifesto, in which Pabst affirms the restorative power of cinema, which, in essence, consists of an incessant dialectic between cut and reunion. With its proper aesthetic means, the cinema can thus repair the after-effects of war.

\section{Keywords}

Pabst (Georg Wilhelm) • Deutscher Film • Neue Sachlichkeit • Film- und Medienästhetik • Rezeptionsgeschichte

Pabst (Georg Wilhelm) • cinéma allemand $•$ Nouvelle Objectivité • esthétique du cinéma $\bullet$ histoire de la réception

Pabst (Georg Wilhelm) • German film $•$ New Objectivity $\bullet$ aesthetics of film $\bullet$ history of reception

L'année cinématographique 2019 a remis Georg Wilhelm Pabst à l'honneur sur les écrans français. La Cinémathèque française lui a en effet consacré une rétrospective du 30 octobre au 25 novembre ${ }^{1}$. On pouvait y revoir ses films les plus connus, comme Loulou (1929) ou L'Opéra de quat'sous (1931), mais aussi y remarquer, avec d'autant plus de lucidité que nous sortions de quatre années de commémorations, qu'il est l'un des seuls cinéastes allemands à avoir traité de la

1 Cet événement fut accompagné de la sortie d'un coffret de treize DVD chez le distributeur Tamasa. Plusieurs articles et émissions ont été consacrés à Pabst (voir par exemple "Pabst ou "l'enfer des femmes" » dans l'émission Mauvais Genres sur France Culture le 29 février 2020).
Première Guerre mondiale dès les années $1920^{2}$. L'événement à la fois artistique et commercial qu'a été cette rétrospective est ainsi entré en résonance avec un événement scientifique : la publication du dossier « Expression artistique et cicatrices de la Première Guerre mondiale. Perspectives franco-allemandes », dont la réalisation reflète la volonté de se laisser l'espace du contretemps scientifique par rapport aux commémorations de 2018.

2 Sur la représentation de la Première Guerre mondiale au cinéma, on pourra consulter les travaux de Laurent Véray, notamment La Grande Guerre au cinéma (2008), qui propose une synthèse grand public des travaux universitaires menés par ce chercheur. 
De tous les réalisateurs qui ont fait la gloire cinématographique de l'Allemagne dans l'entre-deux-guerres, Pabst est le seul à s'être intéressé en profondeur à la Première Guerre mondiale et, plus spécifiquement, aux aspects franco-allemands du conflit. II faut dire que la déclaration de guerre en 1914 surprend Pabst alors qu'il est en France (où il restera interné jusqu'en 1919). Les films qui abordent, plus ou moins directement, la question de la Première Guerre mondiale sont remarquables par leur diversité ${ }^{3}$. L'Amour de Jeanne Ney', drame muet qui mélange étrangement expressionnisme et réalisme, raconte une histoire d'amour entre Paris et la Crimée en 1917 sur fond de révolution bolchévique. Quatre de l'infanterie ${ }^{5}$ et La Tragédie de la mine ${ }^{6}$, qui ont contribué à imposer Pabst comme l'un des chefs de file de la Nouvelle Objectivité cinématographique, frappent tous deux par leur esthétique quasi documentaire et leur engagement pacifiste. Quatre de l'infanterie, adapté du roman d'Ernst Johannsen Vier von der Infanterie (1929), retrace les derniers jours au front de quatre soldats allemands : Karl, l'étudiant, le Bavarois et le lieutenant. La Tragédie de la mine s'inspire de l'accident minier de Courrières en 1906 dont l'action est significativement transposée dans l'entre-deux-guerres : des mineurs allemands organisent une opération de sauvetage de mineurs français emprisonnés sous terre après une explosion. Enfin, Mademoiselle Docteur ${ }^{7}$, production française de divertissement à l'ambition esthétique plus modeste, imagine la dernière mission de la célèbre espionne ${ }^{8}$.

À travers cette hétérogénéité de ton et de style se dessine toutefois une vision cohérente : la guerre est monstrueuse car elle sépare ce qui, dans l'ordre naturel des choses, devrait être uni. Elle est une blessure qui laisse de profondes cicatrices - psychologiques, géographiques et politiques. Le cinéma de Pabst documente ces cicatrices, en même temps qu'il essaie de les réparer.

\section{LA GUERRE COMME BLESSURE}

\section{Une blessure biographique?}

G. W. Pabst est né en 1885 en Bohême de parents viennois. À l'aube de sa carrière artistique, il se tourne d'abord vers le théâtre. II fait ses débuts en tant que comédien en Suisse

3 II est important d'insister sur la diversité (peut-être même l'hétérogénéité) de l'œuvre de Pabst. En France, il est souvent associé à l'expressionnisme. Le Trésor (1923), La Rue de Pabst. En France, il est souvent associé à l'expressionnisme. Le Trésor (1923), La Rue
sans joie (1925) et Loulou avaient ainsi été présentés à la Cinémathèque française dans le cadre de la rétrospective « $F$. W. Murnau et le cinéma expressionniste allemand » (du 26 octobre au 31 décembre 2006). En réalité, l'œuvre de Pabst est traversée par une grand diversité de courants, d'influences et de thèmes. «Ainsi porté par le temps, quand les plus grands portent leur temps, Pabst est un contemporain passif. II suit. Expressionnisme, naturalisme, sexualisme, freudisme, internationalisme, antinazisme, exotisme, nazisme, dénazfication, mysticisme, agnosticisme, toutes les étapes franchies par sa nation, par sa classe, se retrouvent dans sa carrière d'artiste. Ce n'est pas dire qu'il fut homme sans foi. Tout au contraire. Mais, perméable, trop irrésolu pour les trouver en lui, il tenait sa foi et sa force du courant. " (Amengual $1966: 16$ )

4 Die Liebe der Jeanne Ney, Allemagne, 100 mn, 1927.

5 Westfront 1918, Allemagne, $93 \mathrm{mn}, 1930$.

6 Kameradschaft / La Tragédie de la mine, Allemagne / France, 93 mn, 1931

7 Mademoiselle Docteur (aussi connu sous le titre Salonique, nid d'espions), France $116 \mathrm{mn}, 1936$

8 Elsbeth Schragmüller (1887-1940), scientifique allemande, qui a travaillé comme espionne pendant la Première Guerre mondiale. avant de se diriger vers Salzbourg puis Berlin. En 1910, il émigre aux États-Unis et travaille pour le théâtre allemand de New York. En ce début de siècle, « la perméabilité des frontières politiques s'ajoutant à une certaine universalité de la langue allemande, sa carrière s'ouvr[e] sous le signe du cosmopolitisme » (Amengual 1966 : 11). À l'été 1914, Pabst retourne en Allemagne afin de recruter de nouveaux comédiens pour son théâtre. II fait escale en France au moment de la déclaration de guerre et sera donc contraint d'y rester. Il est interné dans le camp de l'île-Longue ${ }^{9}$. Pabst s'implique dans l'organisation d'un théâtre, qui devient le centre de la vie culturelle déjà particulièrement intense dans ce camp, où ont été internés de nombreux autres artistes et intellectuels. Le dessinateur Leo Primavesi conçoit les décors des pièces montées par Pabst, bientôt assisté de Max Pretzfelder, qui arrive au camp fin 1917 - et qui continuera de travailler comme costumier pour Pabst une fois que celui-ci se sera tourné vers le cinéma. Dans l'immédiat après-guerre, Pabst prend la direction de la Neue Wiener Bühne avant d'en venir définitivement au cinéma avec Le Trésor. Les dix années suivantes représentent sa période artistique la plus fructueuse, durant laquelle il tourne les œuvres qui le feront reconnaître par ses contemporains comme un grand cinéaste. II révèle de grandes actrices : Greta Garbo, qui tient l'un de ses premiers rôles dans La Rue sans joie (1925) auprès d'Asta Nielsen (alors star établie du cinéma muet), et bien sûr Louise Brooks, dont il fait une star dans Loulou et $L e$ Journal d'une fille perdue (1929) ${ }^{10}$. Puis sa " trilogie sociale » (Quatre de l'infanterie, La Tragédie de la mine et L'Opéra de quat'sous) contribue à l'imposer non seulement comme un cinéaste mais aussi comme un homme engagé et comme le plus éminent représentant de la Nouvelle Objectivité cinématographique (Amengual $1966: 12$ ).

Il est ainsi tentant d'interpréter la Première Guerre mondiale comme une rupture, si ce n'est une blessure, dans la biographie de Pabst, dont témoignerait le passage d'une forme d'expression artistique à une autre. En effet, l'homme de théâtre qu'était Pabst change de médium après la Première Guerre mondiale et trois des films qui constituent le corpus d'analyse de cet article ont été tournés alors que la carrière de cinéaste de Pabst est à son apogée. À la veille de la Seconde Guerre mondiale, entre 1933 et 1939 c'est-à-dire à l'époque de Mademoiselle Docteur, Pabst vit et travaille en France :

Lorsque la Seconde Guerre mondiale éclata, le 1er septembre 1939, Pabst prévoyait de rejoindre New York à bord d'un bateau italien partant de Rome. Selon sa femme, Pabst se mit à souffrir d'une hernie alors qu'ils se préparaient à quitter Vienne avec leur fils et fut contraint à une longue hospitalisation en Autriche, ce qui le rendit finalement prisonnier des circonstances. Pabst resta dans l'Allemagne nationale-socialiste.

9 Durant la Première Guerre mondiale, des prisonniers étrangers, en particulier allemands, ont été internés dans des camps français. Le plus connu est celui de Garaison (voir la contribution d'Hélène Leclerc dans ce numéro).

10 Pendant toute sa période muette weimarienne, Pabst développe une grande variété de rôles féminins qu'il affranchit des stéréotypes grâce au travail avec les interprètes (Dumas 2018). 
Beaucoup de ses collègues, beaucoup d'historiens et de critiques y ont vu un signe d'opportunisme et Pabst ne retrouva jamais le prestige et le respect dont il jouissait auparavant ${ }^{11}$. (Bock 1990 : 231)

C'est seulement en 1997, à l'occasion de la rétrospective que la Berlinale consacre au cinéaste, que critiques et chercheurs soulignent à quel point le biographisme a entaché la réception critique de Pabst :

Contrairement à ce qui s'est passé pour les autres réalisateurs qui constituent le Panthéon du cinéma muet allemand (Lubitsch, Murnau, Lang), l'accès à l'œuvre de Pabst a jusqu'à présent été soumis aux turbulences qui marquent l'histoire de sa réception. [...] [Le reproche d'avoir collaboré] a fait de l'ombre à la réception des derniers films, certes plus faibles, qu'il a tournés en Autriche, en Italie et en République fédérale - mais a aussi pesé, de manière rétroactive, sur le jugement porté sur ses chefsd'œuvre muets et parlants. (Belach / Bock 1997 : 7)

La rétrospective de Berlin et les publications qui l'accompagnent constituaient une première relecture au-delà du pur biographisme. Près de vingt années après celle de la Berlinale, la rétrospective de la Cinémathèque française entérine la reconnaissance internationale, institutionnelle et critique d'une œuvre dont l'interprétation n'est plus indexée aux seuls atermoiements politiques de son auteur ${ }^{12}$.

\section{Blessures intimes}

Les films que G. W. Pabst a consacrés à la Première Guerre mondiale en dénoncent sans équivoque la monstruosité. La guerre est d'abord un traumatisme sur le plan individuel et intime, ce que les films montrent notamment à travers la destruction des relations amoureuses. Dans Quatre de l'infanterie, Karl a quitté sa famille pour le front. Lorsqu'il rentre en permission, il trouve sa femme au lit avec un autre homme. Au-delà de l'infidélité, il constate surtout qu'une profonde incompréhension règne désormais entre sa femme et lui. Ce n'est pas seulement le contrat de mariage qui a été rompu, mais aussi le lien affectif et émotionnel. Même la bienveillance de la vieille mère de Karl est impuissante à réconcilier les deux époux. En ce qui concerne le plus jeune des quatre soldats, celui qu'on nomme " l'étudiant ", son idylle avec Yvette, la serveuse d'origine française, ne fleurira jamais. Au début du film, leur étreinte dans le grenier est interrompue par des coups de feu, de manière presque anodine. II s'agit pourtant d'un funeste présage : la mort de l'étudiant empêchera les deux amoureux de devenir un couple. Quant aux deux autres films, L'Amour de Jeanne Ney et de Mademoiselle Docteur, la séparation des amants par la 11 Les traductions de citations d'ouvrages en langue étrangère sont toujours les nôtres.
12 Curieusement, la rétrospective de la Cinémathèque constituait ainsi une réponse - si
discrète que personne ne l'a remarquée comme telle - aux controverses liées à « l'affaire
Polanski » qui agitaient au même moment le monde du cinéma et qui posaient la question du rapport entre l'œuvre et l'auteur. guerre en constitue le noyau narratif. Dans sa première partie, L'Amour de Jeanne Ney se pare des atours de la fresque historique, avec des scènes monumentales à l'ambition esthétique et politique. Jeanne est la fille d'un diplomate français qui officie tant bien que mal en Crimée, où il est chargé de faire la chasse aux Bolchéviques pendant la guerre civile russe. Le vieux fonctionnaire est tué par le Bolchévique dont Jeanne, Chimène des temps modernes, est amoureuse. À la suite de cet incident, l'action se déplace alors de la Crimée vers la France : Jeanne doit retourner à Paris pour vivre chez un vieil oncle. Ce retour au pays natal est en réalité un exil pour la jeune femme, déchirée entre son père et son amant, entre la France et la Russie. Dans Mademoiselle Docteur, le capitaine français Carrère flirte avec celle qu'il croit être la journaliste américaine Lillian Stanley et qui est en réalité une espionne allemande. Lorsque le charmant officier découvre la véritable identité de sa fiancée, il doit choisir entre son devoir patriotique et son engagement amoureux. La dernière scène du film est une course-poursuite en voiture d'une grande modernité cinématographique, au terme de laquelle Carrère abat délibérément la femme qu'il aime. La guerre rend les héros, même les plus sympathiques, monstrueux. Elle sépare les couples et elle détruit aussi l'unité de l'âme en la confrontant à d'impossibles choix. Quatre de l'infanterie se termine d'ailleurs sur une scène de délire : l'un des quatre protagonistes, le lieutenant, est devenu fou.

\section{Blessures du paysage}

Quatre de l'infanterie se caractérise par son esthétique très réaliste, ses images brutes, presque documentaires. Pabst place sa caméra à l'orée des tranchées, au ras du sol ; il filme les sillons qui meurtrissent la terre et les barbelés qui déchirent le ciel. À ces blessures visuelles du paysage, Pabst ajoute des blessures sonores. Dans ce paysage ravagé, pas de paix possible : des bruits d'armes et d'explosions retentissent sans cesse, couvrant ou interrompant les conversations des soldats. Quatre de l'infanterie est le premier film parlant de Pabst et l'utilisation qu'il fait du son est virtuose pour l'époque. II en exploite pleinement les possibilités expressives. En l'absence de véritables techniques de mixage, le réalisateur et ses monteur (Hans Oser), ingénieur du son (Karl Brodmerkel) et assistant (Paul Falkenberg) ont déployé des trésors d'ingéniosité pour que les bruits de tirs et d'explosions viennent se superposer aux dialogues des soldats dans les tranchées : entre deux répliques, voire entre deux mots, ils coupaient la pellicule pour insérer à la main des morceaux de bande-son sur lesquels étaient enregistrées des explosions (Geisler 1990 : 93). En outre, les attentes que pouvait avoir un spectateur de l'époque vis-à-vis d'un film parlant sont trompées par la cacophonie de la guerre. Bien loin de laisser libre cours aux conversations des protagonistes, le cinéma parlant de Pabst n'amorce la possibilité d'un dialogue que pour mieux l'anéantir. Le réalisateur se sert ainsi du son pour suggérer formellement la puissance de destruction de la guerre, qui impose ses blessures au paysage mais aussi, très 
concrètement, à la pellicule, elle-même sectionnée pour que soit introduit le bruit des explosions.

\section{Blessures politiques}

L'un des enjeux principaux de la Première Guerre mondiale est celui des frontières et de leur tracé, que ce soit dans l'empire d'Autriche-Hongrie, dans les Balkans ou en Alsace et en Moselle. C'est aussi l'un des principaux enjeux des quatre films de notre corpus d'analyse. Chez Pabst, les frontières politiques, en particulier celles qui sont issues du conflit, séparent arbitrairement les couples (L'Amour de Jeanne Ney, Mademoiselle Docteur), mais surtout des peuples en train de s'éveiller à l'internationalisme (Quatre de l'infanterie, La Tragédie de la mine). Par des moyens tantôt dramatiques et tantôt visuels, Pabst souligne toujours le caractère arbitraire et dérisoire des frontières. Dans Quatre de l'infanterie, les grenades et les balles fusent de tous côtés. Les soldats plaisantent : d'où vient ce tir ? Et celui-là, vers qui est-il dirigé ? Ami, ennemi, peu importe; dans le chaos général, plus rien n'a de sens. Pourtant on continue à se battre. Comme les deux garçonnets qui, au début de la Tragédie de la mine, se disputent à propos d'une ligne de démarcation dans leur jeu de billes, les soldats ne semblent rien faire d'autre sur le champ de bataille que jouer au funeste jeu de la guerre. Pabst met en doute la légitimité des frontières et des lignes de front. Ni l'une ni l'autre ne nourrissent un quelconque sentiment patriotique ; elles ne font que traduire les angoisses métaphysiques à l'aube des catastrophes du $X X^{e}$ siècle, ainsi que le souligne un commentateur de Quatre de l'infanterie :

Cette ligne, qui passe entre la maison d'Yvette, le pays natal et le champ de bataille, est celle du front. Elle ne forge aucunement l'identité en séparant l'ami de l'ennemi. Elle s'inscrit en faux contre l'ordre de la guerre et décrit la ligne d'un combat intérieur, celui du héros mythique en train de perdre la conscience identitaire qu'il a de lui-même. (Kappelhof 1994 : 188)

L'épilogue de La Tragédie de la mine fait écho à la scène d'ouverture avec les deux enfants. La frontière de 1918, que la solidarité des mineurs avait provisoirement abolie, est rétablie. De part et d'autre, garde-frontières français et allemands semblent déguisés et jouent leurs rôles avec une raideur caricaturale. Pour absurde qu'elle soit, la limite n'en est pas moins indéniable, matérialisée très clairement par une grille. Le cinéma de Pabst montre la matérialité des frontières politiques : elles ne sont pas seulement des abstractions mais de véritables cicatrices dans le paysage et la mémoire des peuples.

\section{LE CINÉMA COMME PRINCIPE DE CICATRISATION}

Aux blessures intimes, géographiques et politiques de la guerre, Pabst oppose le cinéma comme principe de cicatrisation. Le cinéma est d'abord un corps de métier où Pabst peut mettre en œuvre, ou plutôt en pratique, l'internationalisme dont il se sent alors proche. La Tragédie de la mine est ainsi une coproduction entre la Nero-Film AG allemande et la Gaumont-Franco-Film Aubert française. Dans Mademoiselle Docteur, production française mettant en scène des stars françaises (Dita Parlo et Louis Jouvet), Pabst fait appel à une équipe transnationale d'artistes et de techniciens avec lesquels il a collaboré tout au long de sa carrière cosmopolite : les scénaristes Irma von Cube et Leo Biriski, le chef-opérateur Eugen Schüfftan, le monteur Mark Sorkin. Le réalisateur se sent investi d'une mission presque sacrée et croit à la puissance de l'art comme outil militant :

Un réalisateur est un combattant. S'il n'est pas un simple artisan qui tourne des films sur commande pour gagner sa croûte. $\mathrm{Si}$, tel le violoniste son instrument ou le peintre ses ustensiles, il conçoit aussi le cinéma comme quelque chose de sacré, alors il pourra, à travers ses films, faire passer ses idées au public, de façon plus ou moins subtile, de la même manière qu'un journaliste politique le fait à travers ses articles. (Pabst 1997 [1933])

Le symbolisme, typique d'une certaine forme de réalisme, de certaines scènes pabstiennes va dans ce sens. Par exemple, à la fin de Quatre de l'infanterie, Karl, blessé, est emmené à l'hôpital. Dans le lit voisin, un soldat français agonisant lui prend la main et balbutie : « Moi pas ennemi, camarade. ». Le geste devient un appel clair et univoque à la réconciliation ; la scène se fait aussi lisible qu'un tract.

Cependant, en regardant les films de Pabst, on s'aperçoit rapidement que le cinéaste ne travaille pas tout à fait « de la même manière qu'un journaliste politique ". Le cinéma n'est pas seulement un médium d'information, mais un art à la puissance à la fois poétique et performative. C'est ainsi, par exemple, que le fait divers dont est inspiré La Tragédie de la mine est transposé par Pabst et ses scénaristes (Peter Martin Lampel, Ladislaus Vajda, Gerbert Rappaport, Karl Otten) de 1906 à l'entre-deux-guerres :

Un accident minier qui s'est produit en 1906 à Courrières, dans le Nord de la France, et lors duquel une petite troupe de mineurs allemands ayant les compétences nécessaires ont assisté leurs collègues français dans un sauvetage de plusieurs jours, allant à l'encontre des idéologies dominantes depuis la victoire allemande de 1871 : ce fait divers offrit à Pabst, dont Quatre de I'infanterie était le premier film à défendre une position politique, un sujet plein de suspense, susceptible d'intéresser un grand public et qui permettait d'adresser un message de réconciliation entre les peuples. Pour la Nero-Film AG de Seymour Nebenzahl, c'était la perspective d'un succès commercial. (Barth $1997: 11)$

Le cinéma, même réaliste, permet de transfigurer la réalité ici de la transposer dans un autre espace-temps. Narration, mais aussi son, image, mise en scène : Pabst utilise toutes les ressources du septième art pour réparer les blessures de la guerre. 
Commençons par le son, dont Pabst fait un usage tout à fait remarquable dès ses premiers films parlants. La Tragédie de la mine est une production franco-allemande qui a été tournée avec des acteurs français et allemands en une unique version bilingue - alors que, en ces premières années du cinéma parlant, l'habitude était de tourner à la chaîne plusieurs versions unilingues d'un même film : on gardait les mêmes décors et les mêmes techniciens mais on changeait les comédiens. À la fin du film, tous les mineurs fêtent le sauvetage réussi des Français par les Allemands. La réconciliation, victoire sur la blessure laissée par la guerre, est entérinée par deux discours, en allemand puis en français, prononcés devant une assemblée de mineurs des deux nationalités. Certes, on peut souligner l'ambivalence de cette séquence, comme l'ont fait les commentateurs les plus attachés aux ambivalences biographiques et personnelles de Pabst : « En présentant ainsi deux discours différents, l'un en français, l'autre en allemand, les divisions nationales que les discours prétendent nier sont en fait reproduites. " (Berman 1990 : 117) On peut toutefois avoir une vision plus poétologique de cette séquence : faire coexister, de manière alors très inhabituelle, l'hétérogénéité de deux langues sur une même bande-son apparaît alors comme un acte fort. II s'agit pour Pabst de réfléchir sur son art et de montrer que, tout comme le cinéma muet, le cinéma parlant est capable d'une certaine universalité par-delà les barrières de la langue.

Cependant, malgré cette utilisation remarquable du son, Pabst croit à la primauté de l'image dans l'art cinématographique :

\footnotetext{
Malgré l'avènement du " parlant », je reste convaincu qu'au cinéma le texte en lui-même reste fort peu de chose. Ce qui compte, c'est l'image. Voilà pourquoi je continue à prétendre que le créateur d'un film est bien plus le metteur en scène que l'auteur du scénario ou les interprètes. (Pabst cité dans Amengual 1966 : 95)
}

L'image a le pouvoir de refermer ces plaies que sont les frontières politiques. Au début de La Tragédie de la mine, la caméra plante le décor : un paysage minier à la frontière franco-allemande, balafré verticalement par les cheminées et poteaux, horizontalement par les fils et barrières, en profondeur par les rails et sillons. Pabst anime ces plans de mouvements de caméra qui franchissent de manière fluide toutes les démarcations présentes dans la zone frontalière ; travellings latéraux et verticaux réunissent en un seul plan le côté allemand et le côté français. Le réalisateur crée ainsi un espace cinématographique commun où circulent librement, à défaut des hommes, références et influences artistiques. Les galeries de La Tragédie de la mine, tout comme les tranchées de Quatre de l'infanterie, forment un contrepoint au réalisme du paysage frontalier et des scènes de front. Elles ouvrent vers un au-delà - ou plutôt vers un en-deçà, un monde souterrain et mystérieux. Les premières sont d'ailleurs un prolongement des secondes, comme le suggère la transposition du fait divers de 1906 dans l'entre-deux-guerres. D'un film à l'autre, un flash-back établit la continuité entre les deux mondes souterrains : alors qu'un mineur allemand déterre un mineur français, des images et des sons des tranchées surgissent à l'écran en même temps que dans la mémoire du Français qui se débat dans les bras de son camarade allemand. La guerre est une cicatrice profonde, certes, mais elle n'en rend que plus belle la solidarité entre mineurs allemands et français. Pabst, héritier du romantisme de Henri de Ofterdingen autant que du réalisme - au demeurant souvent fantastique - de Germinal, filme la mine à la fois comme un microcosme social et comme un univers fantasmagorique, où se mêlent l'eau, la terre et le feu, où fusionnent les contraires, où les peuples se réconcilient par-delà et surtout en-deçà des frontières.

La mise en scène de Pabst, inlassablement, réunit ceux que la guerre a séparés. L'Amour de Jeanne Ney affirme, discrètement mais avec une force qui témoigne d'une inébranlable conviction esthétique, le pouvoir unificateur de l'image cinématographique. Le film préfigure presque les expérimentations formelles de la fin des années 1920 : Pabst filme Paris comme Walther Ruttmann filmera Berlin (Berlin, Symphonie d'une grande ville, 1927) et Dziga Vertov les villes soviétiques (L'Homme à la caméra, 1929). La réunion de Jeanne et du révolutionnaire russe (qui finit par la rejoindre à Paris) donne lieu à de magnifiques scènes d'extérieur. Les retrouvailles des amants se font aux Buttes Chaumont : Jeanne file en voiture le long de la grille, Andrej court au même rythme derrière la rangée d'arbres qui bordent le parc. La caméra les suit de manière assez virtuose, évoquant une définition presque étymologique du cinéma comme "écriture du mouvement ". Au sein même de ce mouvement, les obturations régulières de l'objectif par les arbres ou les barreaux de la grille rappellent les phénakistiscopes, zootropes, praxinoscopes et autres dispositifs du pré-cinéma. De l'intermittence, le cinéma fait continuité. À la fin de cette séquence construite en montage parallèle (la caméra filme tantôt la voiture de Jeanne, tantôt Andrej), lorsque les trajectoires se rejoignent, les deux amants sont aussi réunis à l'image dans un seul et même plan. De manière contemporaine à L'Amour de Jeanne Ney, Dziga Vertov développe dans ses textes théoriques l'idée que le montage est l'opération fondamentale de l'art cinématographique. II est en tout cas à la fois coupe, autrement dit blessure ("Schnitt », dit l'allemand), et réunion (le français " montage " met l'accent sur ce deuxième temps de l'opération). Pabst le cosmopolite réconcilie les étymologies allemande et française, faisant de l'art du montage un art de la cicatrisation.

\section{CONCLUSION}

Il a longtemps été de bon ton dans la critique de qualifier G. W. Pabst de «problématique » (Rentschler 1990) et de déceler dans son œuvre des ambiguïtés à leur tour analysées comme "problématiques ". L'épilogue de La Tragédie de la mine avec les gardes-frontières a ainsi été interprété tantôt comme une critique du nationalisme, tantôt comme une 
attaque nationaliste contre le « Diktat » de Versailles (Kracauer 2019 [1947] : 241). Pourtant, les quatre films de Pabst traitant plus ou moins directement de la Première Guerre mondiale, en sont, sans ambiguitté possible, une dénonciation. Dans Quatre de l'infanterie, La Tragédie de la mine mais aussi dans L'Amour de Jeanne Ney et dans Mademoiselle Docteur, la guerre est une expérience monstrueuse, traumatique, qui blesse l'individu autant que le collectif. Elle sépare ce qui devrait être uni, au premier chef les couples et les peuples. Une autre affirmation se dégage sans équivoque des quatre films : celle du pouvoir qu'a le cinéma, sinon de rétablir cette union, du moins de réparer les blessures de la guerre dans l'ordre du cinéma. Au même titre (ou presque) qu'un tract ou qu'un journal, le cinéma est un médium qui permet de défendre une idée. Mais c'est aussi un art dont la poétique permet de réunir ce qui a été brisé par des moyens tour à tour narratifs, sonores et visuels. De Dziga Vertov à Jean-Luc Godard, nombre de cinéastes-théoriciens ont fait du montage l'opération fondamentale du septième art. Chez Pabst le montage devient, à l'instar du cinéma lui-même, un principe de cicatrisation.

\section{Bibliographie sélective}

Amengual, Barthélémy (1966), Georg Wilhelm Pabst, Paris, Seghers. Atwell, Lee (1977), G. W. Pabst, Boston, Twayne publishers.

Barth, Hermann (1997), « Wie Pabsts Kameradschaft gemacht ist. Eine Drehbuchlektüre », in Barth, Hermann et alii (dir.), Kameradschaft, Munich, Text + Kritik, 10-27.

Belach, Helga / Bock, Hans-Michael (1997), « Editorische Vorbemerkung ", in Barth, Hermann et alii (dir), Kameradschaft, Munich, Text + Kritik, 7-9

Berman, Russel A. (1990), " A Solidarity of Repression » in Rentschler, Eric (dir.), The Films of G. W. Pabst. An Extraterritorial Cinema, New Brunswick / Londres, Rutgers University Press, 116-124.

Bock, Hans-Michael (1990), « Documenting a Life and a Career » in Rentschler, Eric (dir.), The Films of G. W. Pabst. An Extraterritorial Cinema, New Brunswick / Londres, Rutgers University Press, 217-235.

Dumas, Louise (2018), « Les scandaleuses de Weimar. Femmes et actrices dans le cinéma muet de G. W. Pabst », in Positif, n693, 94-97.
Geisler, Michael (1990), "The Battleground of Modernity » in Rentschler, Eric (dir.), The Films of G. W. Pabst. An Extraterritorial Cinema, New Brunswick / Londres, Rutgers University Press, 91-102.

Jacobsen, Wolfgang (1997), G. W. Pabst, Berlin, Stiftung Deutsche Kinemathek, Argon.

Kappelhoff, Hermann (1994), Der möblierte Mensch. G. W. Pabst und die Utopie der Sachlichkeit: ein poetologischer Versuch zum Weimarer Autorenkino, Berlin, Vorwerk 8.

Kracauer, Siegfried (2019 [1947]), From Caligari to Hitler, Quaresima Leonardo (éd.), Princeton / Oxford, Princeton University Press.

Rentschler, Eric (1990), « The Problematic Pabst: An Auteur Directed by History » in Rentschler, Eric (dir.), The Films of G. W. Pabst. An Extraterritorial Cinema, New Brunswick / Londres, Rutgers University Press, 1-23.

Véray, Laurent (2008), La Grande Guerre au cinéma. De la gloire à la mémoire, Paris, Ramsay. 